Research Article

\title{
Peer Review of Manuscripts: A Valuable yet Neglected Educational Tool for Early-Career Researchers
}

\author{
Robert McNair $\mathbb{D}^{1},{ }^{1}$ Hai Anh Le Phuong $\mathbb{D}^{1},{ }^{1}$ Levente Cseri $\mathbb{D}^{1},{ }^{1}$ and Gyorgy Szekely $\mathbb{D}^{1,2}$ \\ ${ }^{1}$ School of Chemical Engineering of Analytical Science, The University of Manchester, The Mill, Sackville Street, \\ Manchester M13 9PL, UK \\ ${ }^{2}$ Advanced Membranes and Porous Materials Center, Physical Science and Engineering Division (PSE), \\ King Abdullah University of Science and Technology (KAUST), Thuwal 23955-6900, Saudi Arabia \\ Correspondence should be addressed to Gyorgy Szekely; gyorgy.szekely@kaust.edu.sa
}

Received 26 January 2019; Accepted 20 May 2019; Published 4 June 2019

Academic Editor: Kirsi Tirri

Copyright (C) 2019 Robert McNair et al. This is an open access article distributed under the Creative Commons Attribution License, which permits unrestricted use, distribution, and reproduction in any medium, provided the original work is properly cited.

\begin{abstract}
With the number of publications being all-time high, academic peer review is imperative to ensure high-quality research content. The wider involvement of postgraduate, early-career researchers (ECRs) has been proposed on several platforms to address the unsustainability of the peer review process caused by a lack of peer reviewers. A survey involving 1203 academics and ECRs in ten countries revealed their attitudes towards the involvement of ECRs in the peer review process. The trends and motives were identified, with emphasis on the peer review being an oft-neglected tool in the skill development of ECRs. In light of the survey results, the transferrable skills that ECRs acquire from performing peer reviews at a crucial stage in their career development are systematically explored. The findings call for further engagement of ECRs in the peer review process under supervisory mentoring.
\end{abstract}

\section{Introduction}

Peer review can be defined as a process where a person reads, checks, and gives their opinion about a piece of work that was written by another scientist or expert working in their same subject area. Generally speaking, two types of peer review can be distinguished: "in-class peer review" for undergraduate students and "academic peer review" for research scholars.

During the in-class peer review, undergraduate students read articles written by fellow classmates and give feedback on their work as part of the taught aspects of the curricula. Academic peer review (hereafter "peer review"), on the contrary, is a more complex process. Peer review is a cornerstone of academic research. In general, the scientific publication process consists of three main participants: the authors submitting a manuscript describing their research, the editors determining whether the manuscript will be sent out for the peer review and eventually accepted for publication, and the peer reviewers performing evaluations, which enable the editor to make a better informed decision about the suitability of the manuscript for publication. These reviewers play an understated yet crucial role in the process, utilising their expertise to constructively critique a piece of work for the benefit of the wider scientific community.

The benefits of the peer review are manyfold. Peer review is essential for maintaining the integrity and credibility of a scientific field. It acts as an effective "filter," spotting manuscripts of insufficient quality and ultimately preventing flawed science from making it to print. Peer review opens up important channels of communication among the authors, editors, and fellow reviewers, allowing all to be heard in a joint contribution to their field. Furthermore, it encourages authors to submit only original and impactful work, improving the quality and efficiency of the publication process.

In a 2013 study on peer review, $90 \%$ of authors felt that the peer review improved the quality of their published paper, with $84 \%$ also stating that it is an essential practice to control scientific communication [1]. In a more recent study, $74 \%$ of authors stated that the quality of their published paper was improved as a result of the peer review, with $82 \%$ stating that there is no control of scientific communication 
without the peer review [2]. Although the importance of the peer review was rated similarly, satisfaction with the quality of peer review decreased by a considerable $16 \%$ over only three years. The reason could be the increasing number of manuscripts submitted each year (Figure 1) to an increasing number of journals.

The 2017 metrics from the abstract and citation database Scopus classified 36,612 sources as journals. Figure 1 displays the number of publications per year from 1978 to 2017. The 20-year period from 1978 to 1997 saw an overall increase of 599,652 publications per year. The subsequent 20 -year period (1998-2017) saw the number of publications almost triple, with an overall increase of 1,691,356 publications per year. The rapid increase in number of publications per year highlights the need for a greater number of skilled peer reviewers to keep pace with the growth of scientific research.

One telling statistic revealed that more than a quarter of all authors agreed that the peer review was unsustainable due to a lack of willing peer reviewers. Typically, peer review opportunities are offered by a journal editor to individuals with expertise in a given field, such as industry professionals or established academics at higher education institutions who have been previously published by the journal. These experts are frequently forced to decline opportunities to the peer review, with $57 \%$ of those surveyed stating that the main reason for declining the peer review is due to generally being too busy or other work commitments [2].

To address the distinct lack of reviewers, recent efforts have been made to innovate the peer review process. Certain efforts have focused on expanding the reviewer pool by encouraging the peer review by early-career researchers (ECRs). In fact, concerns have been raised that excluding ECRs from the peer review could lead to their marginalisation in the scientific community and a lack of diversity in the thought processes of reviewers [3]. ECRs such as $\mathrm{PhD}$ candidates and postdoctoral researchers have been underrepresented among peer reviewers, but they have a plethora of skills to offer and the potential to acquire more by participating in the peer review process (Figure 2). ECRs often possess more time than their academic supervisors, a focused understanding of their subject area and a keen willingness to broaden their existing knowledge. These attributes make them an excellent solution to the shortage of peer reviewers.

The Reviewer Recognition Platform is an initiative specifically geared towards increasing the participation of ECRs in the peer review. This platform allows lessestablished reviewers to volunteer their expertise on a given field, specifying preferences and areas of research. In this system, if a manuscript submitted to a journal matches their areas of expertise, then the "volunpeers" are alerted and may "book" the article that they wish to review. After the editor makes a final decision on the manuscript, personalised feedback may be exchanged. This pilot has already been launched by established journals, with promising initial results. The pilot has received an editor satisfaction of $80 \%$ and a reviewer satisfaction of $100 \%$ [4].

The American Chemical Society (ACS) also offers an online peer-review training course for scientific researchers.

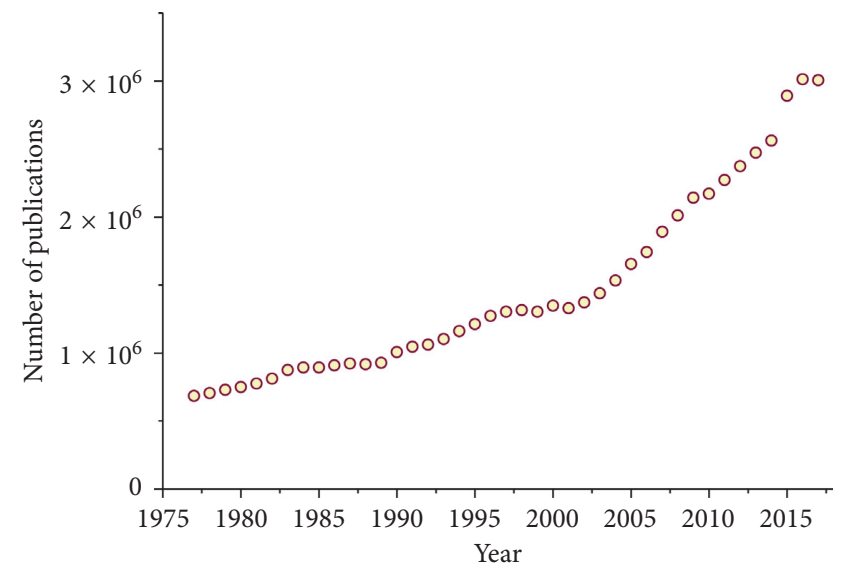

FIGURE 1: The increasing number of publications per year over the last 40 years (1978-2017, Source: Web of Science).

The ACS Reviewer Lab course is designed jointly by editors and researchers, comprising modules on ethical guidelines, literature searching, critical thinking, and academic writing skills for reviewers with little or no previous experience of handling academic manuscripts [5]. Sense about Science, a UK charity dedicated to tackling the misrepresentation of scientific evidence, released an article describing the "nuts and bolts" of peer review [6], using interviews with academics and editors to draft guidelines on what ECRs can expect from the peer review process.

These initiatives demonstrate not only the willingness of ECRs to engage in the peer review but also the necessity of training this new generation of peer reviewers. ECRs conducting peer review are given the opportunity to make valuable contributions to their scientific field, while enhancing various areas of their skills set in the process. Our study draws on the experiences of both academics and ECRs to assess how peer review can influence some of these skills at a crucial stage in their career development. A multinational survey involving both academics and ECRs was performed to identify the trends and motives regarding the involvement of ECRs in the peer review process. The transferrable skills that ECRs can acquire were systematically explored to reveal the full educational potential of wellconducted peer reviewing.

\section{Methodology}

To gain insights into attitudes towards the involvement of ECRs in the peer review, we circulated a survey to academics in ten countries across the globe. The survey covered various aspects of the peer review process, including how many review invitations are typically accepted by academics, whether they involved ECRs (postgraduate researchers only) in their reviews, and if so, then what was their motivation for the involvement of ECRs. We requested that the academics evaluate the impact of the peer review on the integral skills of their students, based on their previous experience. Moreover, a separate survey was circulated to ECRs (the student survey), to determine their attitudes towards involvement in the peer review. Full details of the survey questions for both 


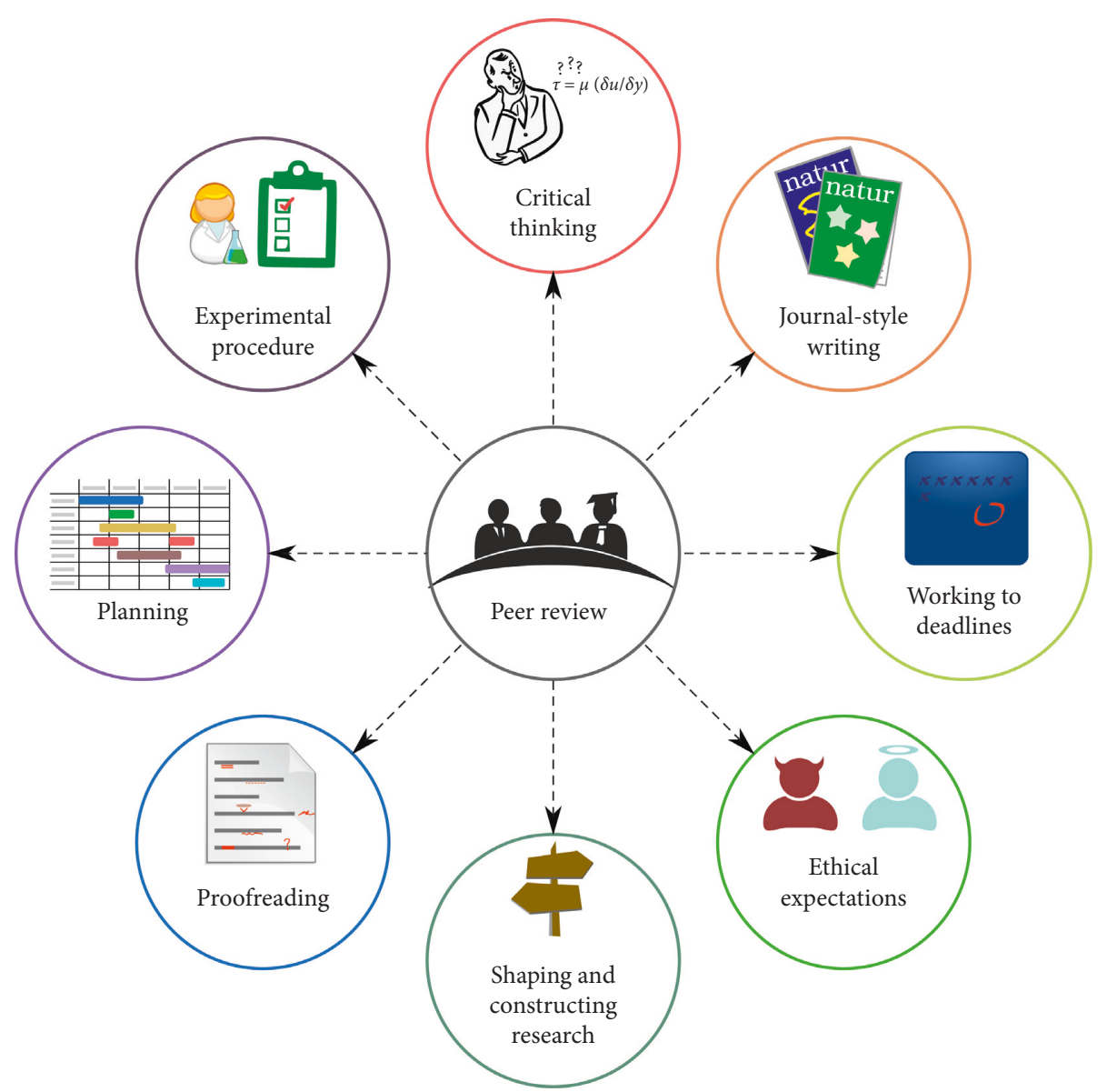

Figure 2: Overview of professional skills needed for ECRs that can be impacted by peer-reviewing manuscripts.

the academic and ECR surveys can be found in Supplementary Materials. For the purposes of the survey, the terminology postgraduate students (PGRs) were used instead of ECRs (Supplementary Materials). This was to clearly differentiate from undergraduate researchers, who were not the subject of our study.

\section{Results and Discussion}

3.1. Current Attitudes towards the Involvement of EarlyCareer Researchers in Peer Review. The surveys revealed that the average number of peer review invitations accepted per month ranged from 2.7 (Germany) to 4.9 (China and Hong Kong), with a mean value of 3.5 accepted reviews per month across all of the surveyed countries (Figure 3). The percentage of academics who were assisted in their peer reviews by ECRs ranged from 27\% (China and Hong Kong) to $67 \%$ (UK). We note that only $27 \%$ of the academics from China and Hong Kong offered peer review opportunities to ECRs, despite accepting the most peer review invitations. These results were somewhat unexpected, as a higher number of accepted reviews could be expected to result in a higher tendency to offer peer review opportunities. For example, this trend is evident in Figure 3, where an increase in accepted reviews from Germany to UK generally corresponds to a higher percentage of reviews assisted by ECRs.
The data suggest a marked reluctance of academics to offer peer review opportunities to ECRs in China and Hong Kong compared to the other surveyed countries. Taking the mean value over all ten countries, only 55\% of all academic respondents offered peer review opportunities to ECRs under their supervision. Similar results were obtained from the student survey; $42 \%$ of all ECRs had never been invited to peer review manuscripts (Figure S8). These initial findings suggest significant room for growth in the involvement of ECRs in the peer review process.

Only $47 \%$ of academics who accepted $1-3$ reviews per month (Figure 4(a)) were assisted by ECRs in the peer preview process, while $66 \%$ of respondents who accepted 4 or more manuscripts for the peer review were assisted by ECRs. Academics who accepted fewer reviews per month offered peer review to ECRs mainly for "teaching" purposes (46\%) rather than "work-efficiency" purposes (1\%) (Figure 4; Section S2.2). In contrast, respondents who accepted more reviews were much more likely to invite ECRs to participate due to "work-efficiency" purposes (35\%).

The survey data indicate a current lack of emphasis placed on the use of peer review for the professional skill development of ECRs, in particular for academics who accepted a large amount of peer review invitations. This finding was further underscored by the ECR survey, where only $31 \%$ of ECRs reported receiving constructive feedback 


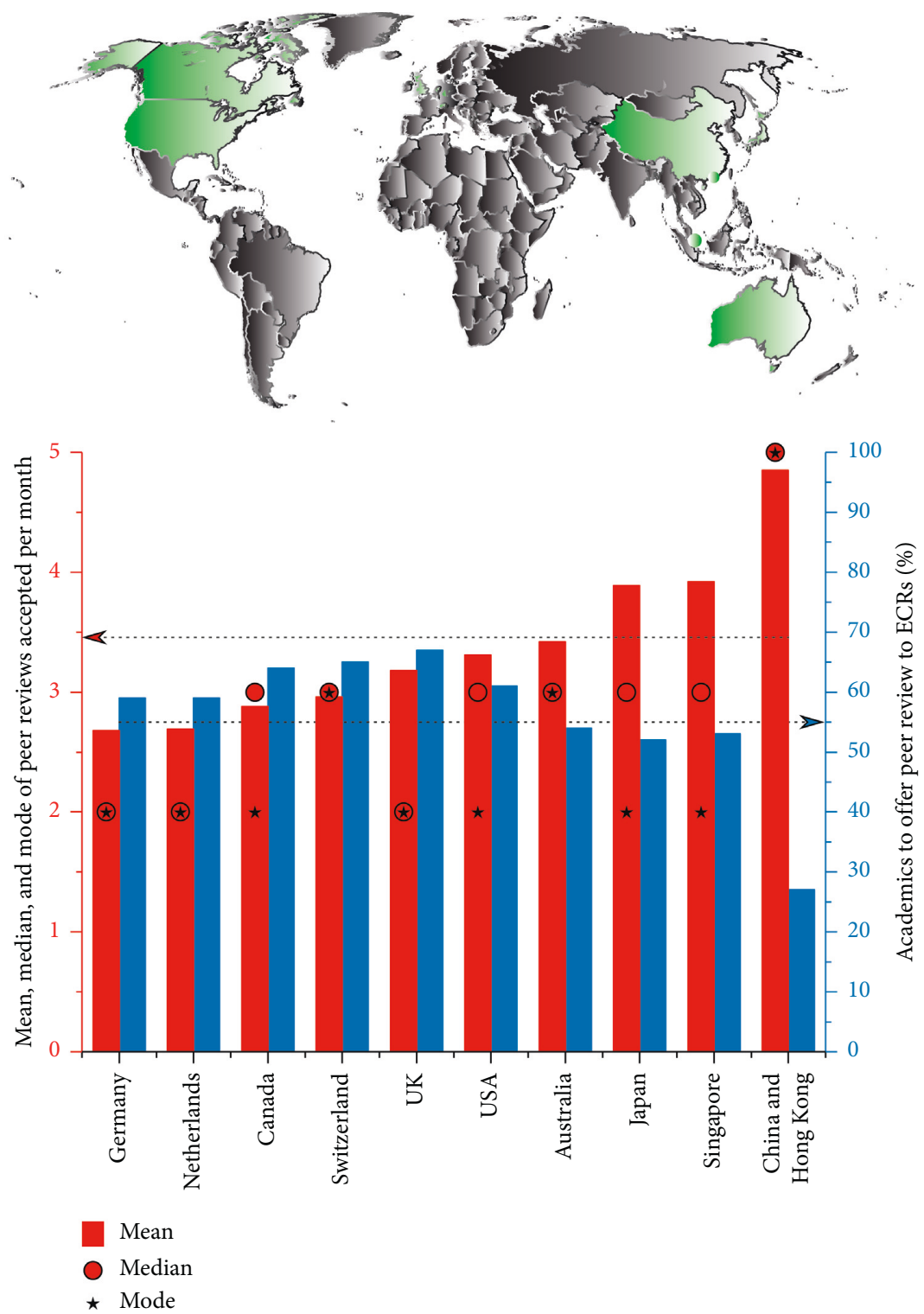

Figure 3: Mean, median, and mode of peer reviews accepted per month by academics (left axis (red)) and percentage of academics who offered peer review opportunities to ECRs (right axis (blue)) for all countries represented in the survey. The total average of monthly accepted peer reviews and the overall percentage of peer review offering academics are indicated by the dotted lines.

from their supervisors regarding the quality of their review (Section S3.8). Constructive feedback is essential for effective learning [7], and peer review as an educational tool is no exception. These findings show that the engagement of ECRs in the peer review process is seen by academics as a necessity, and it is not fully appreciated or practiced as a valuable educational tool.

3.2. Enhancing Skills: The Power of Peer Review. To establish the effectiveness of the peer review as an educational tool, academics and ECRs were requested to score the "educational value" of the peer review in terms of eight skills often used by ECRs. Both groups were asked to score peer review's impact on the skills using a scale of $0-10$, with 0 indicating that the peer review adds no educational value to the given skill and 10 representing the maximum educational value. Figure 5 displays both the mean educational value scores and the number of responses for each score and each skill; the colour of the box corresponds to the numbers given in the scale bar. The mean scores for individual countries can be found in Figure S4. The full breakdown of both the academic and ECR surveys are detailed in Supplementary Materials. The responses from academics and ECRs, as well as the influence that the peer review can have on these eight skills, are discussed in the subsequent sections.

3.2.1. Critical Thinking. Critical thinking plays an essential role in everyday life as well as in all aspects of research, from data interpretation and literature reviewing to hypothesis evaluation and paradigm changes. Oxford Dictionaries 


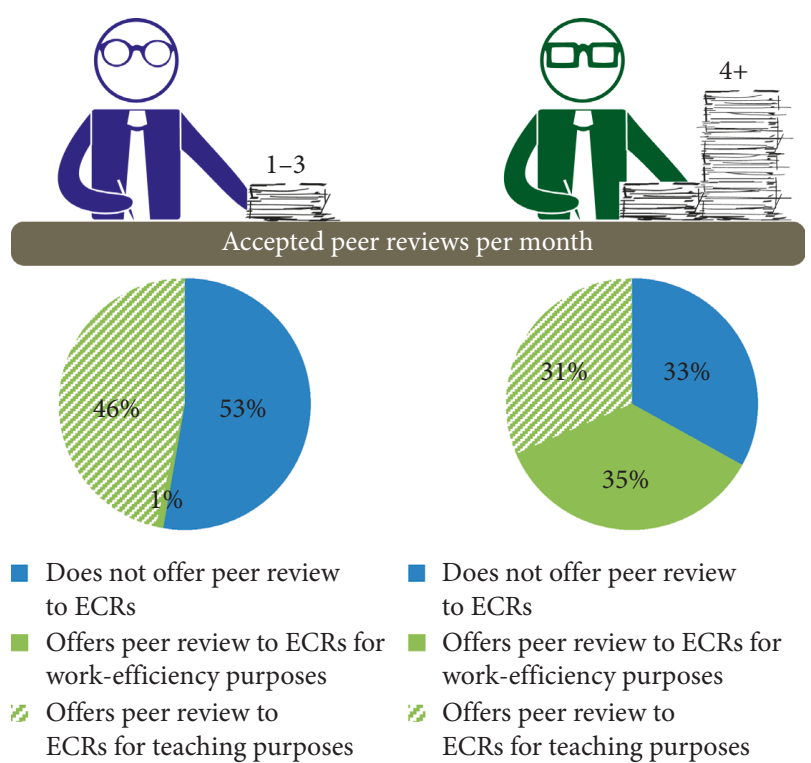

(a)

(b)

FIGURE 4: Statistics on academics accepting and sharing peer reviews with ECRs: percentage of academics who offered (green) and who did not offer peer review opportunities to ECRs (blue). Solid green fill signifies the percentage of respondents who offered peer review for "work-efficiency" oriented purposes, while the striped fill shows the percentage of respondents who offered peer review for "teaching" oriented purposes. Panels (a) and (b) display results for respondents who accept $1-3$ and $4+$ peer reviews per month, respectively.

define critical thinking as "the objective analysis and evaluation of an issue in order to form a judgement." It involves being self-guided, self-disciplined, and self-reflective, whilst also being aware of other mindsets [8]. In research, errors that could be avoided by critical thinking include incorrect or superficial interpretation of the literature, solid conclusions drawn from partial or unreliable experimental data, overlooking equally fitting explanations, and accepting or refuting hypotheses without sufficient evidence. Nonetheless, standard education systems place little emphasis on the development of critical thinking although teacher evaluations and peer assessments have recently drawn increasing interest [9]. Peer reviewers are responsible for judging the novelty and validity of a work before recommending its acceptance or rejection. To make that final decision, critical thinking is required. Thus, peer reviewing helps ECRs be aware of, develop an eye for, and avoid errors that could undermine the scientific integrity of their own work.

The feedback received via our survey from both academics and ECRs provides a strong basis for the use of peer review as an educational tool for the development of critical thinking. Out of all eight skills listed in the survey, critical thinking was judged by both academics and ECRs to benefit the most from the peer review (Figure 5 and Figure S10). The mean educational value added by the peer review to critical thinking skills was scored at $8.8 / 10$ and $6.8 / 10$ by academics and ECRs, respectively (a full comparison of the scores is provided in Section S3.5). The outstanding potential of the peer review is reinforced by the $96 \%$ of academic respondents who scored its educational value in terms of critical thinking within the range of 7-10 (Figure S7).

3.2.2. Journal-Style Writing. An attainable goal for ECRs is to see a piece of their own work published in a peer-reviewed journal. However, for this to occur, the principal requirement is that the ECR must be able to convey their hypotheses and results in a way that can be understood by a broad scientific community. Various works have been published that affirm the importance of academic or "journal-style" writing for ECRs and outline the crucial skills they should look to perfect when preparing a manuscript $[10,11]$. Furthermore, methods that actively improve the academic writing skills of students have been practiced for decades, such as peer assessment workshops [12], language classes, and the incorporation of academic writing into taught curricula [13].

From a scientific standpoint, a key aspect of journal-style writing is preparing manuscript content (figures, text, and data tables) that is information-rich and maximises the research output in the most concise way. By reviewing manuscripts submitted to different journals, ECRs can become proficient in spotting content that is reliable and impactful and, just as importantly, content that does not meet the criteria for submission to a peer-reviewed journal. Examples of the latter include duplicated figures and text, "space-filling text" that describes irrelevant information, ambiguous data that are not suitably reinforced by conclusions in the text, informal expressions, superlatives, scattered information, lack of building arguments, and insufficient captions. Improvement in the scientific writing skills of ECRs comes from the experience of reading and practice. Peer reviewing teaches ECRs to recognize succinct and effective scientific presentation, as the manuscripts showcase both good and bad aspects of journal-style writing.

As with critical thinking, the overwhelming response from academics was that peer review had a strong positive effect on the journal-style writing of ECRs; the mean educational value was $8.5 / 10$, and $92 \%$ of respondents scored the educational value between 7 and 10 (Figure S7). Behind critical thinking only, journal-style writing was scored by ECRs as the skill which was influenced most positively by the peer review, receiving a mean score of 6.7/10. Again, these findings reinforce the use of the peer review for the enhancement of journal-style writing skills in ECRs.

3.2.3. Proofreading. Proofreading involves the act of rereading and rewriting a piece of work by focusing on and correcting grammar, punctuations, and spellings [14]. Proofreading, however, not only allows for the correction of small typos and grammatical errors but also provides an opportunity to judge the work and to improve its clarity and readability.

Proofreading is often considered a tedious task that is known to be important yet often neglected. As a result, small mistakes and typos may remain within the submitted manuscript; these could be meaningless or may significantly alter 

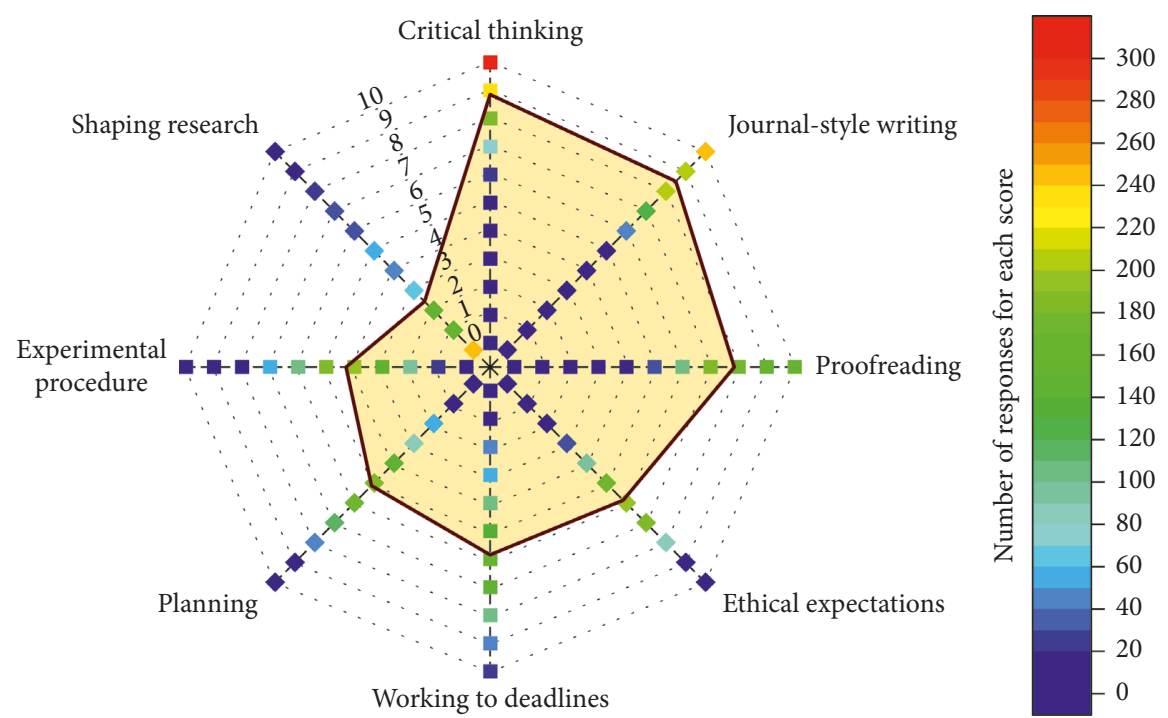

FIGURE 5: Statistics for the educational value of peer review (0-10) towards 8 skills needed for ECRs (yellow fill: mean scores; scale bar: number of responses for each score $0-10)$.

the scientific message. Whilst these errors should not affect the judgements of reviewers regarding the novelty and quality of manuscripts, they could negatively impact the paper and even result in the loss of the author's credibility as a reliable researcher [15]. In fact, poorly written and edited manuscripts correlate with a 'high rejection rate' [16]. The research contained in a manuscript could be flawless and promising; however, upon encountering many avoidable mistakes, a reviewer may begin to question the quality of work. For example, "How can I trust the validity of results if the manuscript is full of mistakes?" or "Is the methodology and work trustworthy if the authors did not make the effort to reread the manuscript even once?" To address this, reviewers may use the "Halo Effect" concept, to avoid drawing assumptions on a piece of research based on these avoidable errors [17].

By allowing ECRs to identify errors in other works, proofreading manuscripts help to ensure that these are not replicated in their own reports and become aware of how these small mistakes could result in loss of credibility. Nevertheless, a reviewer must not give in unconscious bias; a paper should not be rejected solely because the manuscript was written by a nonnative English speaker. Instead, reviewers should focus mainly on the research itself to provide equal opportunity for every researcher [18].

Proofreading was ranked by both academics and ECRs as the skill third was most improved by the peer review (Figure 5). Proofreading received a mean educational value score of 7.8/10 from academics and 6.4/10 from ECRs. However, the distribution of responses was larger than those of critical thinking and journal-style writing; the responses ranged from 2 to 10 for academics (Figure S7) and from 3 to 10 for ECRs (Figure S11). This may indicate a slight tendency of ECRs to neglect proofreading compared to the aforementioned skills.

3.2.4. Ethical Expectations: Conflicts of Interest and Confidentiality. Ethical expectations and obligations of reviewers are unavoidable subjects in the discussion of the peer review that brings with them differing opinions. Similar to other aspects of peer reviewing, research students rarely handle confidential documents during their studies. However, it is an important part of any research position, whether in academia or industry. As manuscripts sent for the peer review are confidential, general control measures must be taken regarding their storage, printing, and disposal. Moreover, manuscripts submitted to journals contain by their very nature new and unpublished scientific theories, methods, and results. It is of utmost importance for the integrity of the peer review process that the reviewer does not use or share any of the ideas contained in a manuscript they are reviewing. For example, ECRs will eventually review a paper closely related to their own research, and some unpublished information in the manuscript could help them in their research project. However, novel information and new ideas from the reviewed paper must not be used in any way at that stage. Only after publication may the ECR build on and reference the paper.

Through the peer review process under supervised conditions, ECRs can learn what research integrity and confidentiality means in practice. Additional to the reviewer guides provided by journals, the Committee on Publication Ethics (COPE) published a detailed guideline that outlines several ethical aspects of the peer review and establishes good reviewer practices [19]. According to the COPE guidelines, the involvement of ECRs in the review process is not only allowed but also encouraged as part of training and mentoring.

The differing views from academics on the subject are reflected in the survey findings. The distribution of responses from academics spanned the whole scoring range (Figure S7), resulting in a mean educational value score of 5.8/10. Indeed, some academics felt that this issue should be raised during the survey, stating that it was considered inherently unethical to share an unpublished manuscript with ECRs. However, most journals allow the involvement of 
ECRs in the peer review process, subject to editorial approval. In this case, the involved ECR's name and affiliation should be provided not only for procedural transparency but also to give the ECR appropriate credit for their work [20]. Ethical expectations were rated slightly higher by ECRs (5.9) than academics (5.8) although the difference was not statistically significant. This indicated that ECRs may find peer review beneficial to their awareness of ethical guidelines. Interestingly, only $34 \%$ of all ECR survey respondents who had practiced the peer review said they were given detailed instructions about confidentiality by their supervisor (Section S3.7). This low percentage suggests that many ECRs are conducting the peer review without being given detailed instructions about its ethical aspects, which reinforce concerns about sharing manuscripts with ECRs. It also highlights the need for an open discussion between academics and ECRs in which academics provide peer review guidelines, allowing ECRs to better appreciate ethical and confidentiality issues. Offering peer review to ECRs ingrains into them the ethical practices of both reviewing and publishing from an early stage in their careers.

3.2.5. Working to Deadlines. Everyone is given deadlines during their lifetime, whether for homework, school assignments, or work for a client. Deadlines, both self-imposed and external, are important for completing projects, achieving personal goals, and learning time management. In fact, deadlines, especially those in the near future, have been shown to enhance motivation to finish tasks and to decrease procrastination [21]. However, ECRs rarely face strict deadlines during their studies or work since only internal progress reports need to be submitted. By agreeing to assist in a peer review, an ECR agrees to provide a fair and constructive review within a given timeframe. Thus, the peer review provides an opportunity for ECRs to experience working to an external deadline.

Some academics left comments stating that including less-experienced ECRs in the peer review lengthens the process. Reviewing a manuscript is time-consuming, in particular for inexperienced ECRs. The thorough evaluation of a manuscript requires several read-throughs and full consideration of all data, methods, hypotheses, and results. However, as an ECR conducts more peer reviews and gains familiarity with the timings of the publication process, they will become more efficient and aware of how a delayed review could also delay the publication of an article. Reviewers must also balance the workload and responsibilities of the peer review with their own research tasks, maintaining a high standard of both requires proficiency in meeting impending deadlines. Reviewing manuscripts whilst simultaneously carrying out research gives ECRs the opportunity to learn prioritisation techniques.

"Working to deadlines" (5.8/10) was the skill that displayed the broadest distribution of responses in the academic survey (Figure S7); all educational values 0-10 received a response. On the contrary, $20 \%$ of all academic respondents rated the educational value of the skill in the range $8-10$. The responses to the ECR survey displayed the same broad distribution (Figure S11) and an even lower mean educational value score of $5.1 / 10$. These inconsistent results confirm the mixed experiences of academics and ECRs alike regarding the benefit of the peer review to meet deadlines in general.

3.2.6. Planning. Planning is an essential aspect of workload and time management and an inescapable necessity in all graduate study. Studies have shown that the ability of a student to manage their time can correlate directly to their academic achievements [22]. Various projects have sought to improve the time management skills of ECRs. A study by Flinders University implemented behavioural coaching as a tool to affect long-term attitude changes [23]. This study claimed reduced negative behaviours such as procrastination, lower levels of stress, and improved time management by the participants.

Peer review can provide invaluable experience for ECRs in terms of both experiment and manuscript preparation. From an experimental standpoint, ECRs learn by analysing manuscripts to prioritise tasks and set experimental timelines. ECRs also learn the structure of a manuscript and how to carefully plan the content of each section, e.g., a thorough review of the relevant literature in Introduction that verifies the novelty of their work [24] and, in Methods, sufficient descriptions of the determining measurement parameters and material quantities and purities for reproducibility. Crucially, planning prevents ECRs from wasting time on trivial factors and improves their overall work efficiency. Reducing detrimental attributes, such as task aversion and uncertainty, in ECRs can help to improve their organisational skills and may result in higher academic performance. Time is a precious commodity for ECRs, and time invested in the effective peer review could ultimately save days or weeks over the course of their research.

The educational value of planning was scored $5 / 10$ by academics; this middle-of-the-road score suggests an overall neutral opinion. On the contrary, planning did show a broad distribution among the academic survey responses (0-9). ECRs scored the educational value of peer review towards planning (5.4/10) higher than academics (5.0/10) with a statistically significant difference. This highlights the potential of the peer review as an educational tool in the view of ECRs.

3.2.7. Experimental Procedure. Whilst peer review requires no hands-on experimental work, it can indirectly teach ECRs valuable experimental protocol, which can be transferred into their own work. Before the postgraduate study, many ECRs have little knowledge of the true nature of full-time research. Peer reviewing a manuscript can give insights into the duration, order, and frequency of experiments that should be carried out in order to produce a journal-quality piece of work. Reviewing the systematic practices contained in submitted manuscripts can give inexperienced reviewers an idea of experimental design, which will become crucial during the progression of their research careers. In addition, peer review exposes ECRs to many different experimental 
reports related to their field. ECRs are introduced to good practice, i.e., behaviours such as reliable data gathering, reporting both accuracies and errors in results, and assessing the validity of methods used to prove hypotheses. Again, peer review allows ECRs to thoroughly scrutinise experimental work done by their peers.

The educational value of the peer review for an experimental procedure was scored $4.2 / 10$ and $3.6 / 10$ by academics and ECRs, respectively. These initial findings suggest that neither academics nor ECRs found the peer review very beneficial to their existing experimental skills.

3.2.8. Shaping Research. The phrase "shaping research" in the context of the peer review could be construed in different ways by academics and ECRs alike. One interpretation could be that the respondents understood the question to mean "shaping research" by using a novel piece of unpublished experimental work or replicating the details of a study. Such actions are not "skills" to be inherited from peer review; rather, they are behaviours that must be discouraged and penalised to maintain the integrity of publishing within the scientific community.

Instead, a second interpretation of the question is that the peer review gives ECRs the opportunity to positively shape their research partly by learning the established techniques (e.g., characterisations and testing methods) described in a manuscript, but primarily by learning how to recognise the novelty, impact, and originality of the work. ECRs may develop this skill through repeated exposure to and scrutiny of the scientific data and conclusions drawn in manuscripts being reviewed. During the review process, a valuable dialogue opens among the reviewers, authors, and editors. The work described in a manuscript must be able to withstand scrutiny from reviewers, and the authors must be willing to adapt and revise their manuscript based on the comments of peers within their field [25]. The experience of the peer review teaches ECRs the impactful themes used to measure the value of a work against similar literature. Once the ECRs are aware of these themes, they are able to consistently improve their own work so that it merits publication in a scholarly journal. Academics and ECRs rated the educational value of peer review at 2.3/10 and 2.2/10, respectively.

\section{Conclusions}

Our study, for the first time, analysed the participation of early-career researchers (ECRs) in the process of peer review and evaluated the impact of their participation on integral skills. From the survey responses of 1203 academics and ECRs across ten countries, we gained valuable insights into current attitudes towards ECR involvement in the peer review. We found that ECRs are generally underutilised in the peer review and that the peer review is not exploited fully as an educational tool. Our academic survey found that $45 \%$ of all respondents had not offered peer review opportunities to ECRs. These results were mirrored by the ECR survey results; $42 \%$ of all ECR respondents had never been invited to the peer review. Based on these findings, academics and journal must strive to provide greater opportunities for ECRs to conduct scientific peer reviews. In addition, greater emphasis should be placed on the existing resources available to ECRs, such as peer-review training courses offered by journals. Complementary to this, our Protocol for ECRs to Effectively Review (PEER, Section 4 in Supplementary Materials) provides useful guidelines that ECRs may follow when conducting preliminary reviews, including important questions to consider on different aspects of a manuscript. Our survey found that academics accept many peer review invitations per month (4+), and only $31 \%$ offered the peer review to ECRs for teaching-related purposes. Furthermore, we found that only $31 \%$ of all ECRs who had conducted reviews received constructive feedback from their supervisors about the quality of their review. These statistics highlight the importance of mentoring and guidance for ECRs when conducting peer reviews; this may come from academics, journal editors, or any experienced reviewer. The constructive feedback that ECRs receive will establish areas of strength and weakness when carrying out reviews, facilitating professional skills development and increasing the quality and efficiency of the review. According to the survey responses, academics and ECRs agree that critical thinking, journal-style writing, and proofreading are the top three skills that benefit from participating in the peer review. Furthermore, our study highlighted several other essential skills for ECRs that have the potential to be enhanced through the peer review. Aiding the expansion of these skills in ECRs will help to develop innovative, ethical, and driven researchers, which will benefit scientific communities worldwide.

\section{Data Availability}

The data and survey findings used to support the findings of this study are included within the file in Supplementary Materials.

\section{Conflicts of Interest}

The authors declare that there are no conflicts of interest.

\section{Supplementary Materials}

Section 1 outlines the methodology, detailing the questions selected for both academic and ECR surveys. Both qualitative and quantitative (statistical) analyses of the individual questions are subsequently provided. Section 2 outlines the responses to the academic survey. Section 3 provides an analysis of the responses to the student survey and draws comparisons between the two surveys. Section 4 outlines PEER guidelines. (Supplementary Materials)

\section{References}

[1] Wiley RANO Survey, Principles of Peer Review, Wiley Author Services, 2013, https://authorservices.wiley.com/asset/Principles_ of_Peer_Review.pdf. 
[2] Elsevier, The Publishing Research Consortium (PRC) Survey, How Researchers Really Feel About Peer Review, Elsevier, Amsterdam, Netherlands, 2016, https://www.elsevier.com/ data/assets/pdf_file/0004/205717/PeerReviewWeek2016_Info graphic.pdf.

[3] G. McDowell, Early Career Researchers and their Involvement in Peer Review, ASAPbio, 2018, http://asapbio.org/mcdowell-ecrs.

[4] C. Tancock, Innovation in Peer Review: Introducing 'Volunpeers', Elsevier, Amsterdam, Netherlands, 2018, https:// www.elsevier.com/connect/reviewers-update/innovation-inpeer-review-introducing-volunpeers.

[5] ACS Reviewer LabTM, "Peer-review training for scientific researchers," ACS Publications, Washington, DC, USA, 2018, https://www.acsreviewerlab.org/?utm_source=Reviewer \%20Email\&utm_medium=Email\&utm_campaign=Mentor\%20 Angle_Est\%20Reviewers.

[6] J. Wilson, Peer Review-The Nuts and Bolts (A Guide for EarlyCareer Researchers), Sense about Science, 2012, http:// senseaboutscience.org/wp-content/uploads/2016/09/peer-reviewthe-nuts-and-bolts.pdf.

[7] M. N. Ovando, "Constructive feedback: a key to successful teaching and learning," International Journal of Educational Management, vol. 8, no. 6, pp. 19-22, 1994.

[8] N. Sieroka, V. I. Otto, and G. Folkers, "Critical thinking in education and research-why and how?," Angewandte Chemie International Edition, vol. 57, no. 51, pp. 16574-16575, 2018.

[9] D. Morrison, How to Promote Critical Thinking with Online Discussion Forums, Online Learning Insights, 2013, https:// onlinelearninginsights.wordpress.com/2013/10/01/how-topromote-critical-thinking-with-online-discussion-forums/.

[10] P. J. Silvia, How to Write a Lot: A Practical Guide to Productive Academic Writing, American Psychological Association, Worcester, MS, USA, 2018.

[11] J. M. Swales and C. B. Feak, Academic Writing for Graduate Students: Essential Tasks and Skills, University of Michigan Press, Ann Arbor, MI, USA, 2004.

[12] K. J. Topping, E. F. Smith, I. Swanson, and A. Elliot, "Formative peer assessment of academic writing between postgraduate students," Assessment \& Evaluation in Higher Education, vol. 25, no. 2, pp. 149-169, 2000.

[13] C. Coffin, M. J. Curry, S. Goodman, A. Hewings, T. M. Lillis, and J. Swann, Teaching Academic Writing: A Toolkit for Higher Education, Routledge, London, UK, 2003.

[14] J. Madraso, "Proofreading: the skill we've neglected to teach," English Journal, vol. 82, pp. 32-41, 1993.

[15] A. Wallwork, English for Writing Research Papers, Springer, New York, NY, USA, 2011.

[16] R. Coates, B. Sturgeon, J. Bohannan, and E. Pasini, "Language and publication in "cardiovascular research" articles," Cardiovascular Research, vol. 53, no. 2, pp. 279-285, 2002.

[17] P. Olk and P. Rosenzweig, "The halo effect and the challenge of management inquiry: a dialog between Phil Rosenzweig and Paul Olk," Journal of Management Inquiry, vol. 19, no. 1, pp. 48-54, 2010.

[18] A. J. Herrera, "Language bias discredits the peer-review system," Nature, vol. 397, p. 467, 1999.

[19] Committee on Publication Ethics (COPE), Ethical Guidelines for Peer Reviewers, 2017, http://www.publicationethics.org.

[20] J. van Harten, A Reviewer's Guide to Ethics in Publishing, Elsevier, Amsterdam, Netherlands, 2015, https://www.elsevier.com/ reviewers-update/story/publishing-ethics/a-reviewers-guideto-publishing-ethics.

[21] S. van Etten, M. Pressley, G. Freebern, and M. Echevarria, "An interview study of college freshmens' beliefs about their academic motivation," European Journal of Psychology of Education, vol. 13, no. 1, pp. 105-130, 1998.

[22] S. N. A. M. Razali, M. S. Rusiman, W. S. Gan, and N. Arbin, "The impact of time management on students' academic achievement," Journal of Physics Conference Series, vol. 995, article 012042, 2018.

[23] H. Kearns, M. Gardiner, and K. Marshall, "Innovation in $\mathrm{PhD}$ completion: the hardy shall succeed (and be happy!)," Higher Education Research \& Development, vol. 27, no. 1, pp. 77-89, 2008.

[24] L. R. Zientek, J. M. Werner, M. V. Campuzano, and K. Nimon, "The use of Google scholar for research and research dissemination," New Horizons in Adult Education \& Human Resource Development, vol. 30, no. 1, pp. 39-46, 2018.

[25] Editorial, "The art of the revision," Nature Chemical Biology, vol. 7, no. 1, p. 1, 2011. 


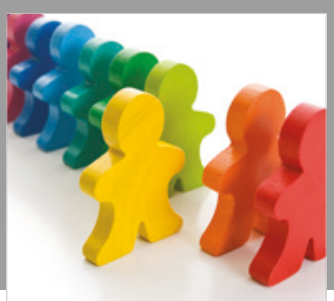

Autism

Research and Treatment
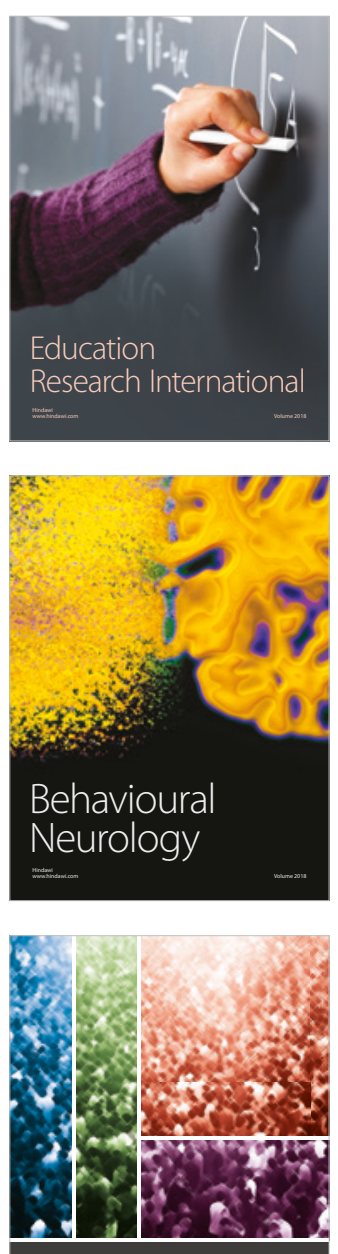

International Journal of

Population Research

$\underline{-m}$

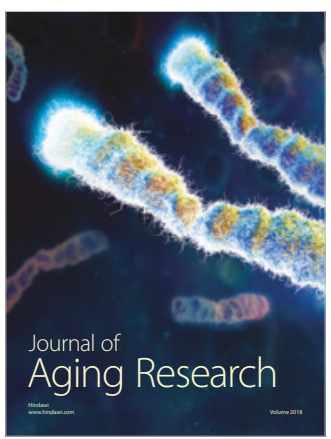

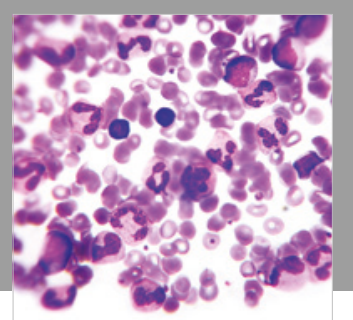

Pathology

Research International$$
=
$$

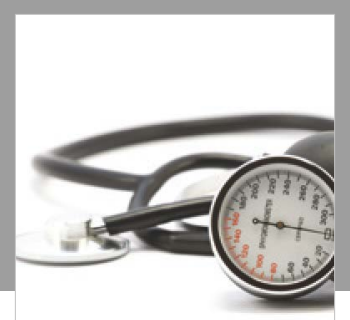

Nursing

Research and Practice

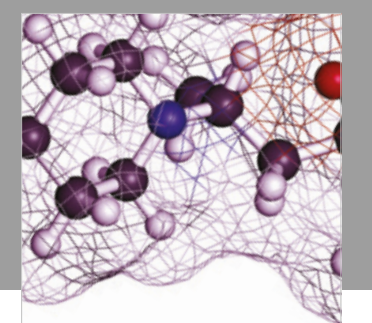

Pain

Research and Management

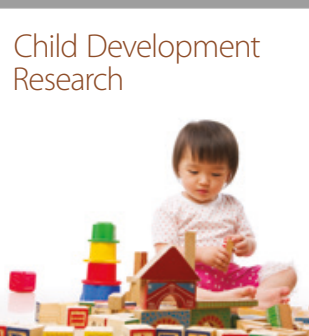

बाD

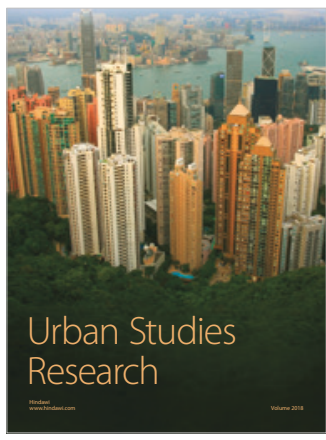

\section{Hindawi}

Submit your manuscripts at

www.hindawi.com
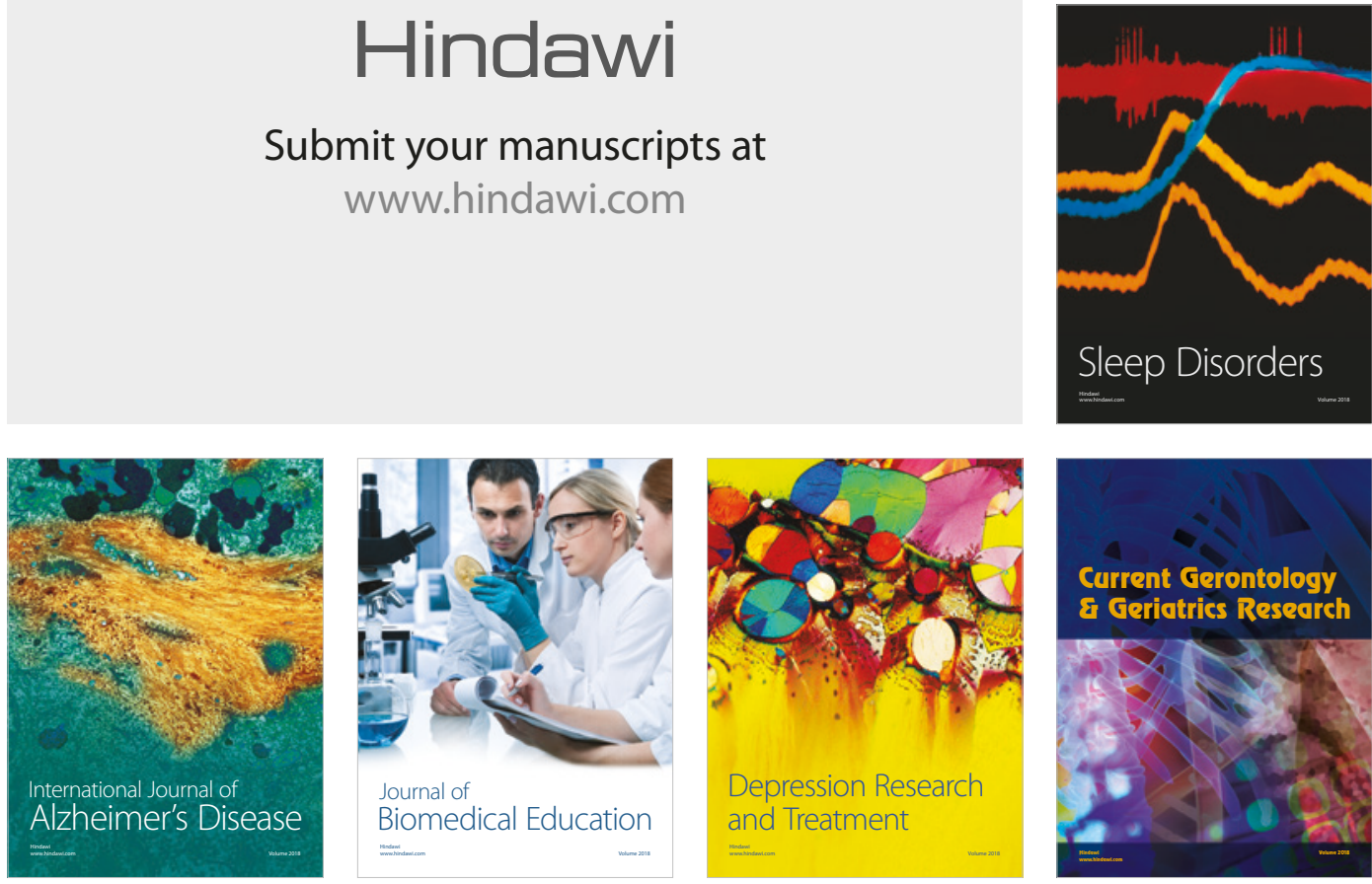

Journal of

Biomedical Education

$=$

smman

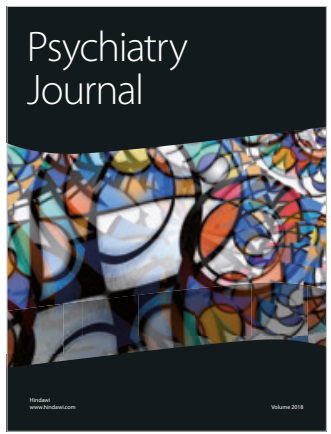

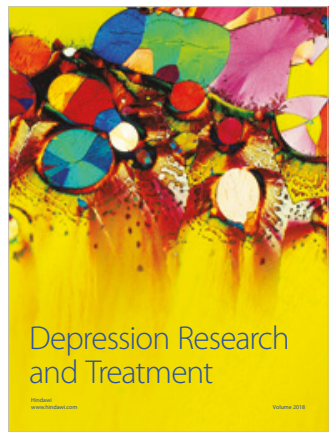
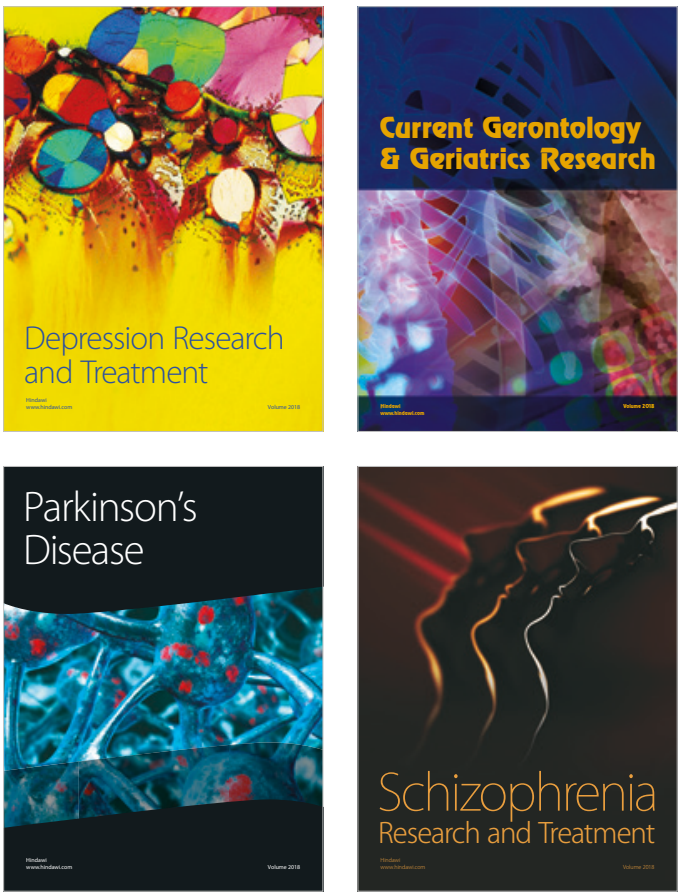